\title{
Serum pentraxin 3 is a novel marker in Crohn's disease
}

\author{
JINGBO CHEN*, XIAOLIN XU*, LIJIAN XIA, XIANGPENG XI, BO LIU and MINGYU YANG \\ Department of Gastrointestinal Surgery, Qianfoshan Hospital of Shandong Province, \\ Jinan, Shandong 250014, P.R. China
}

Received February 18, 2014; Accepted December 3, 2014

DOI: $10.3892 / \mathrm{mmr} .2015 .3451$

\begin{abstract}
In Crohn's disease (CD), clinical symptoms correspond poorly to inflammatory disease activity. Biomarkers, which reflect the level of intestinal inflammation are considered to enable effective monitoring of disease activity. The present study evaluated the role of serum pentraxin 3 (PTX3) as a marker of disease activity in patients with CD. Serum PTX3 levels were determined using an enzyme-linked immunosorbent assay in patients with $\mathrm{CD}(\mathrm{n}=240)$, ulcerative colitis (UC; $\mathrm{n}=240)$ and healthy controls $(\mathrm{HC} ; \mathrm{n}=80)$. The expression levels of PTX3 were also evaluated in 35 fresh colonic tissue samples by western blot analysis. The association between levels of serum PTX3 with disease activity and its diagnostic role were analyzed. The levels of serum PTX3 were significantly increased in patients with active $\mathrm{CD}$ compared with patients in remission ( $\mathrm{UC}$ and $\mathrm{HC}$; $\mathrm{P}<0.01$ ). A significant increase in the expression levels of PTX3 was identified at a protein level in inflamed colonic tissues compared with uninflamed colonic tissues. The levels of serum PTX3 were also positively correlated with disease activity in $\mathrm{CD}$. These results indicated that serum and colonic expression of PTX3 were upregulated in patients with active $\mathrm{CD}$, suggesting serum PTX3 is superior to CRP in predicting CD activity.
\end{abstract}

\section{Introduction}

Inflammatory bowel disease (IBD) is a chronic intestinal disorder comprising Crohn's disease (CD) and ulcerative colitis (UC). The major clinical features of IBD include diarrhea, rectal bleeding, abdominal pain and malnutrition (1). The prevalence of IBD has markedly increased in recent years in China and other countries $(2,3)$, which has significantly affected the quality of life of the affected patients. Currently, the diagnosis and assessment of IBD relies predominantly on a combination of taking

Correspondence to: Dr Mingyu Yang, Department of Gastrointestinal Surgery, Qianfoshan Hospital of Shandong Province, 1676610 Run Road, Jinan, Shandong 250014, P.R. China E-mail: mingyu_yang@yeah.net

*Contributed equally

Key words: Crohn's disease, pentraxin 3, disease activity, marker a patient history and physical examination in association with laboratory, endoscopic, histological and radiographic investigations. In order to reliably diagnose IBD, specific biomarkers that may reflect the activity and progression of this disease are required $(2,4)$. In addition, the identification of potential biomarkers has the potential to improve current understanding of the pathogenesis of the disease and provide novel therapeutic targets.

Pentraxin 3 (PTX3) has been established as a multimeric inflammatory mediator, which shares structural homology with hepatic short pentraxins, including $\mathrm{C}$-reactive protein (CRP) and the serum amyloid $\mathrm{P}$ component $(5,6)$. PTX3 is produced at sites of inflammation and is considered to identify inflammatory disease more effectively than CRP $(7,8)$. However, the putative role of PTX3 in the development of CD remains to be fully elucidated. The current study aimed to investigate the potential of PTX3 as a novel biomarker of CD.

\section{Materials and methods}

Patients and samples. The present, single-center, study was performed between 2007 and 2009 at the Department of Gastrointestinal surgery, Qianfoshan Hospital of Shandong Province (Jinan, China). The diagnoses of CD and UC were based on conventional clinical, radiological, endoscopic and histopathological observations (9). Serum was collected from all the patients prior to electronic colonoscopy $(n=240)$ and biopsy samples were obtained from the patients with $\mathrm{CD}$ in order to confirm diagnosis. The controls included age and gender-matched patients with UC $(n=240)$ and healthy volunteers $(n=80)$. The patient characteristics are shown in Tables I and II.

The clinical activity was determined using the Crohn's Disease Activity Index (CDAI). A CDAI $\geq 150$ was defined as the active phase of the disease and a CDAI $<150$ was defined as remission. Written informed consent was obtained from all the individuals and all procedures involving human subjects were approved by the Institutional Review Boards and ethics committee of Qianfoshan Hospital of Shandong Province.

Determination of serum PTX3 levels. Human serum PTX3 levels were detected using the Human Pentraxin 3/TSG-14 Quantikine enzyme-linked immunosorbent assay (ELISA) kit (R\&D Systems, Inc., Minneapolis, MN, USA). The ELISAs were performed in triplicate, according to the manufacturer's instructions. 
Table I. Patient characteristics of each group.

\begin{tabular}{lcccc}
\hline Group & $\begin{array}{c}\text { Active CD } \\
(\mathrm{n}=139)\end{array}$ & $\begin{array}{c}\text { CD in remission } \\
(\mathrm{n}=101)\end{array}$ & $\begin{array}{c}\text { UC } \\
(\mathrm{n}=240)\end{array}$ & $\begin{array}{c}\text { CRP-negative subgroup } \\
(\mathrm{n}=143)\end{array}$ \\
\hline Male/female ratio & $76 / 63$ & $50 / 51$ & $130 / 110$ & $75 / 68$ \\
Age (years) & $36.29 \pm 10.32$ & $35.18 \pm 13.46$ & $37.47 \pm 15.36$ & $37.45 \pm 13.98$ \\
Weight (kg) & $59.14 \pm 12.63$ & $62.80 \pm 13.61$ & $61.44 \pm 12.29$ & $58.62 \pm 11.83$ \\
Height $(\mathrm{cm})$ & $164.09 \pm 10.42$ & $166.71 \pm 11.15$ & $165.23 \pm 9.78$ & $165.60 \pm 11.81$ \\
\hline
\end{tabular}

$\mathrm{CD}$, Crohn's disease; UC, ulcerative colitis; CRP, C-reactive protein.

Table II. Disease distribution.

\begin{tabular}{lrr} 
Distribution & $(\mathrm{n}=139)$ & $(\mathrm{n}=101)$ \\
\hline Isolated ileal & 21 & 16 \\
Isolated colonic & 27 & 13 \\
Isolated ileocolonic & 42 & 17 \\
Isolated anoperineal & 9 & 20 \\
Anoperineal associated with another site & 35 & 28 \\
Ileal, colonic or ileocolonic associated with another non-anoperineal site & 5 & 7
\end{tabular}

$\mathrm{CD}$, Crohn's disease.

Western blot analysis. The colonic tissue samples, which were frozen in liquid nitrogen (Qingdao Ruifeng Gas Co., Ltd., Qingdao, China), were lysed in cell lysis buffer (Sigma-Aldrich, St. Louis, MO, USA) containing $20 \mathrm{mM}$ Tris- $\mathrm{HCl}$ (pH 7.5), $150 \mathrm{mM} \mathrm{NaCl}, 1 \mathrm{mM} \mathrm{Na}{ }_{2}$ EDTA, $1 \mathrm{mM}$ ethylene glycol tetraacetic acid, $1 \%$ Triton, $2.5 \mathrm{mM}$ sodium pyrophosphate, $1 \mathrm{mM} \beta$-glycerophosphate, $1 \mathrm{mM} \mathrm{Na}_{3} \mathrm{VO}_{4}$ and $1 \mu \mathrm{g} / \mathrm{ml}$ leupeptin. The samples were homogenized using a polytron tissue grinder (CH-6010; Kinematica, Luzern, Switzerland) at $4^{\circ} \mathrm{C}$ and then cleared by centrifugation at $15,000 \mathrm{x}$ g for $30 \mathrm{~min}$ at $4^{\circ} \mathrm{C}$ and the protein concentration was determined using a Bicinchonic Acid kit (Pierce Biotechnology, Inc., Rockford, IL, USA). The total proteins from each sample $(50 \mu \mathrm{g})$ were heated at $95^{\circ} \mathrm{C}$ for 5 min subsequent to mixing with an equal volume of 2x SDS loading buffer (Cyagen Bioscience Inc., Guangzhou, China). The samples were separated using 12\% SDS-polyacrylamide gel electrophoresis gels (Cyagen Bioscience Inc.) and electrotransferred to polyvinylidene fluoride membranes (EMD Millipore, Billerica, MA, USA). The membrane was blocked in Tris-buffered saline with Tween 20 (TBS-T; Cyagen Bioscience Inc.) containing 5\% skimmed milk (Cyagen Bioscience Inc.) at room temperature for $2 \mathrm{~h}$. The membranes were then incubated with the anti-mouse monoclonal anti-PTX3 antibody (1:2,000; sc-358922; Santa Cruz Biotechnology, Inc., Dallas, TX, USA) in TBS-T overnight at $4^{\circ} \mathrm{C}$. Subsequent to washing with TBS-T, the membranes were incubated in 5\% skimmed milk in TBS-T buffer containing a rabbit anti-mouse monoclonal immunoglobulin G secondary antibody (1:5,000; sc-365062Santa Cruz Biotechnology, Inc.) for $60 \mathrm{~min}$ at room temperature with agitation (B11-1, Shanghai Sile Co., Shanghai, China). PTX3 was detected using a Chemiluminescent Reagent
Plus kit (Perkin-Elmer Life Sciences, Santa Clara, CA, USA). Normalization of the protein loading was performed using the mouse monoclonal anti-GAPDH housekeeping control antibody (1:5,000; sc-365062; Santa Cruz Biotechnology, Inc.).

Statistical analysis. Statistical analysis was performed using SPSS software, version 16.0 (SPSS, Inc., Chicago, IL, USA) and the Kolmogorov-Smirnov test was used to analyze the data for normality. The data are presented as either the mean \pm standard deviation or the median \pm interquartile range if continuous and as ' $n$ ' if categorical. Differences between two groups were analyzed using the Mann-Whitney U test. Spearman's/Pearson correlation analysis was used to measure the correlation between the serum PTX3 levels and the CDAI. Receiver operating characteristic (ROC) curves and the area under the ROC curve (AUC) were calculated. $\mathrm{P}<0.05$ was considered to indicate a statistically significant difference.

\section{Results}

Increased expression of PTX3 in patients with active $C D$. Serum PTX3 levels were evaluated using ELISA and were significantly increased in patients with active CD compared with the control patients with UC $(621.98 \pm 178.09$, vs. 410 $.40 \pm 106.34 \mathrm{ng} / \mathrm{ml} ; \mathrm{P}<0.001)$. A significant difference was also observed between the serum PTX3 levels in patients with active $\mathrm{CD}$ compared with those with $\mathrm{CD}$ in remission $(621.98 \pm 178.09$ vs. $379.72 \pm 93.21 \mathrm{ng} / \mathrm{ml}$; P<0.001; Fig. 1$)$. To further confirm these results, the serum levels of PTX3 were determined in 80 healthy controls. The serum PTX3 levels 


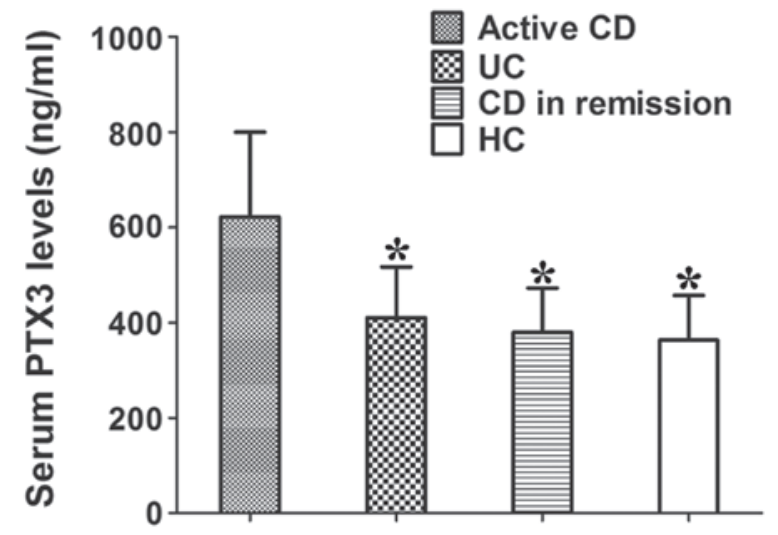

Figure 1. Levels of serum PTX3 in the active CD ( $n=139)$, UC $(n=240), C D$ in remission $(\mathrm{n}=101)$ and $\mathrm{HC}(\mathrm{n}=80)$ groups. ${ }^{*} \mathrm{P}<0.001$, vs. active CD. PTX3, pentraxin 3; CD, Crohn's disease; UC, ulcerative colitis; HC, healthy control.
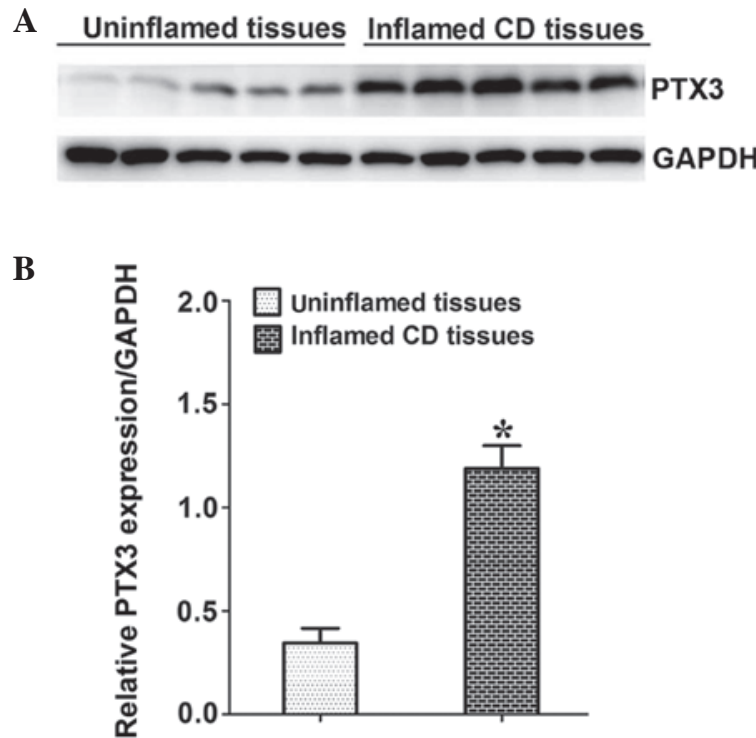

Figure 2. Expression levels of PTX3 were increased in inflamed CD colonic tissues. (A) Representative western blots of five paired fresh colon tissue samples in inflamed and uninflamed sites. The expression levels of PTX3 were increased in the inflamed sites compared with the uninflamed colonic tissues. (B) Western blot analysis of band density in 35 fresh colon tissue samples demonstrated that the relative expression levels of PTX3 were significantly increased in the inflamed CD tissues compared with the uninflamed tissues. Values are presented as the mean \pm standard error of the mean. ${ }^{*} \mathrm{P}<0.001$ vs. uninflamed tissue. PTX3, pentraxin 3; CD, Crohn's disease.

were significantly increased in the patients with active CD compared with the healthy controls $(621.98 \pm 178.09$ vs. 362.9 $7 \pm 94.32$ ng/ml; P<0.001; Fig. 1).

In addition, the expression levels of PTX3 were examined in 35 recently resected inflamed colonic tissues vs. uninflamed tissues from patients with CD using western blot analysis. This confirmed that the expression of PTX3 was significantly higher in the inflamed colonic tissues than in uninflamed sites (Fig. 2). These results suggested that PTX3 was upregulated in active $\mathrm{CD}$ intestinal inflammation.

Serum PTX3 levels are correlated with disease activity in patients with $C D$. The present study also investigated the correlation between serum PTX3 levels and the CDAI in patients
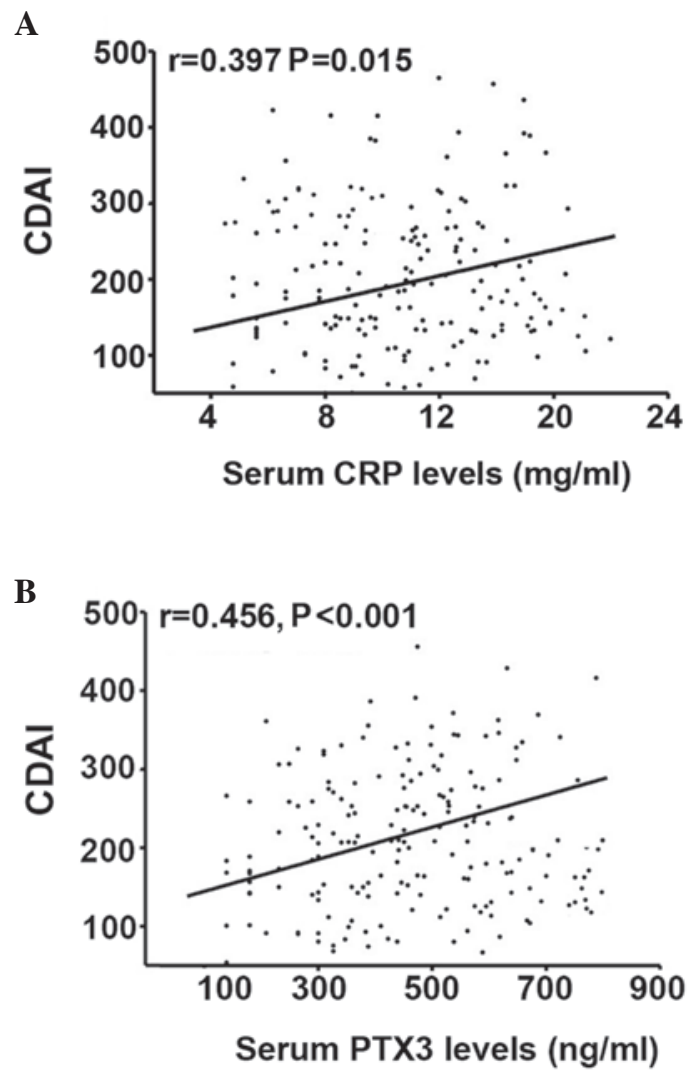

Figure 3. Levels of serum PTX3 are correlated with disease activity in patients with $\mathrm{CD}$. Positive correlations were observed between the (A) level of serum CRP and CDAI and the level of (B) serum PTX3 and CDAI. PTX3, pentraxin 3; CD, Crohn's disease; CRP, C-reactive protein; CDAI, Crohn's Disease Activity Index.

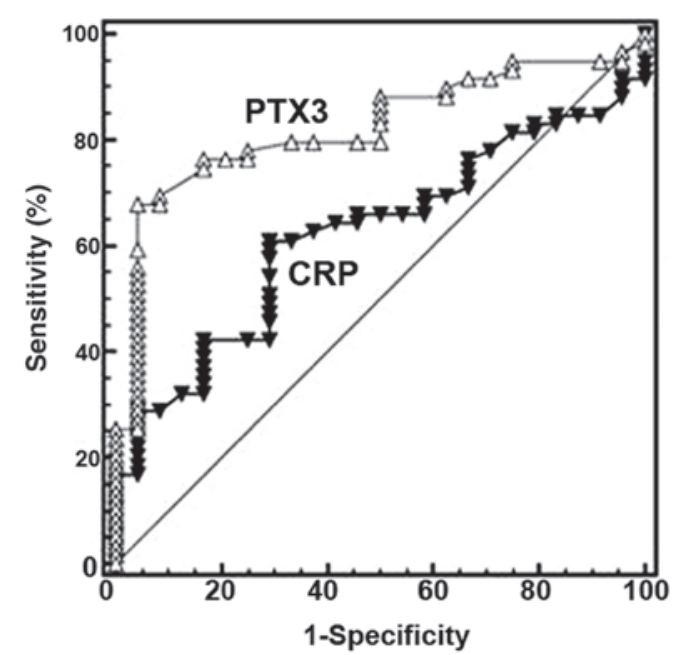

Figure 4. Serum PTX3 as an activity index in patients with CD. The AUC was observed to be 0.85 for serum PTX3 levels and 0.64 for CRP levels. PTX3, pentraxin 3; CD, Crohn's disease; ROC, receiver operating characteristic; AUC, area under the ROC curve; CRP, C-reactive protein.

with $\mathrm{CD}$. A positive correlation was observed between the levels of $\mathrm{CRP}$ and the CDAI ( $\mathrm{r}=0.397 ; \mathrm{P}=0.015 ;$ Fig. $3 \mathrm{~A})$ and between the levels of serum PTX3 and the CDAI $(r=0.456 ; \mathrm{P}<0.001$; Fig. 3B). The serum levels of PTX3 were inversely correlated with the CRP levels in patients with $\mathrm{CD}(\mathrm{r}=-0.62 ; \mathrm{P}<0.0001)$, however, no correlation was observed when a CRP-negative 
subgroup (CRP cutoff value $<4.2 \mathrm{mg} / \mathrm{ml}$ ) was analyzed ( $\mathrm{r}=0.13$; $\mathrm{P}=0.48$ ); data not shown). In this CRP-negative group, the serum PTX3 levels remained significantly correlated with CDAI ( $r=0.416 ; \mathrm{P}=0.026)$, however, no significant correlation was identified between CRP and the CDAI in this subgroup $(r=0.085 ; \mathrm{P}=0.44)$. These observations indicated a more marked correlation between the CDAI and serum PTX3 than serum CRP in CD.

Evaluation of serum PTX3 levels as an activity index in patients with $C D$. By generating an ROC curve, the sensitivity and specificity of serum PTX3, for active CD and CD in remission, as a CDAI were determined (Fig. 4). Cutoff points were determined by the maximum sum of the sensitivity and specificity. The cutoff value of serum PTX3 levels was $506.74 \mathrm{ng} / \mathrm{ml}$ (sensitivity and specificity values of 79.6 and $87.3 \%$, respectively). By contrast, when the cutoff value of CRP levels was set at $4.2 \mathrm{mg} / \mathrm{ml}$, the sensitivity was $61.6 \%$ and the specificity was $68.3 \%$. The AUC for serum PTX3 levels was 0.85, whereas the AUC for CRP levels was 0.64. These results highlight the potential of using serum PTX3 levels as a marker for the evaluation of disease activity in patients with $\mathrm{CD}$.

\section{Discussion}

In clinical practice, currently used inflammatory markers, including CRP and ESR do not provide sufficient accuracy to predict and quantify mucosal inflammation and lesions in CD (10). The present study aimed to evaluate the expression levels and function of PTX3 in CD. The expression levels of serum and colonic PTX3 were found to be significantly upregulated in patients with active $\mathrm{CD}$ compared with the patients with UC and the healthy controls. Additionally, significant correlation was observed between the expression levels of serum PTX3 with disease activity in CD and PTX3 was superior to CRP as a marker for disease activity. These results demonstrated that serum PTX3 is a potential indicator for disease activity in patients with $\mathrm{CD}$.

PTX3 has been identified as an early marker of innate immunity and inflammatory responses and is structurally linked to CRP $(11,12)$. PTX3 belongs to the acute phase class of proteins, of which CRP is also a member, and its concentration has been reported to increase significantly in the early stages of inflammation (13). PTX3 is an essential component of the humoral arm of innate immunity and independently associates with the risk of developing vascular inflammation (14). The plasma PTX3 concentration has been observed to increase significantly during hemodialysis as a rapid and sensitive marker of hemodialysis-induced inflammation (15). A previous study also observed that PTX3 correlated with the severity of sepsis and peaked earlier than CRP in patients with ventilator-associated lung inflammation, demonstrating superior prognostic value in the prediction of mortality (16). Changes in the concentration of PTX3 in the early phase of acute pancreatitis are similar to that of interleukin- 6 and its levels peak at an earlier stage compared with CRP, suggesting that PTX3 may be of value in the early evaluation and in predicting the severity of acute pancreatitis (13). In addition, data from a previous study demonstrated that PTX3 may be a more sensitive marker of the local inflammatory response resulting from vessel injury compared with CRP, and lower levels of PTX3 may reflect potent anti-inflammatory properties (17). In the present study, it was also observed that the correlation between serum PTX3 and CDAI in CD was more marked than that observed with serum CRP.

In conclusion, the present study demonstrated that the expression levels of serum and colonic PTX3 were increased in patients with active $\mathrm{CD}$. These findings suggested that PTX3 may be a more effective potential biomarker of inflammation compared with CRP to predict CD activity. Further investigation is required to clarify the role of PTX3 as an alternative biomarker to CRP in CD.

\section{Acknowledgements}

The present study was supported by the Natural Science Foundation of Shandong Province (no. ZR2011HQ054).

\section{References}

1. Xavier RJ and Podolsky DK: Unravelling the pathogenesis of inflammatory bowel disease. Nature 448: 427-434, 2007.

2. Lewis JD: The utility of biomarkers in the diagnosis and therapy of inflammatory bowel disease. Gastroenterology 140: 1817-1826, 2011.

3. Wang YF, Ouyang Q and Hu RW: Progression of inflammatory bowel disease in China. J Dig Dis 11: 76-82, 2010.

4. Lahiff C, Safaie P, Awais A, et al: The Crohn's disease activity index (CDAI) is similarly elevated in patients with Crohn's disease and in patients with irritable bowel syndrome. Aliment Pharmacol Ther 37: 786-794, 2013.

5. Alles VV, Bottazzi B, Peri G, Golay J, Introna M and Mantovani A: Inducible expression of PTX3, a new member of the pentraxin family, in human mononuclear phagocytes. Blood 84: 3483-3493, 1994.

6. Klouche M, Peri G, Knabbe C, Eckstein HH, Schmid FX, Schmitz G and Mantovani A: Modified atherogenic lipoproteins induce expression of pentraxin-3 by human vascular smooth muscle cells. Atherosclerosis 175: 221-228, 2004.

7. Fazzini F, Peri G, Doni A, et al: PTX3 in small-vessel vasculitides: an independent indicator of disease activity produced at sites of inflammation. Arthritis Rheum 44: 2841-2850, 2001.

8. Baldini M, Maugeri N, Ramirez GA, et al: Selective up-regulation of the soluble pattern-recognition receptor pentraxin 3 and of vascular endothelial growth factor in giant cell arteritis: relevance for recent optic nerve ischemia. Arthritis Rheum 64: 854-865, 2012.

9. Lennard-Jones JE: Classification of inflammatory bowel disease. Scand J Gastroenterol Suppl 170: 2-6, 1989.

10. Benitez JM, Meuwis MA, Reenaers C, Van Kemseke C, Meunier P and Louis E: Role of endoscopy, cross-sectional imaging and biomarkers in Crohn's disease monitoring. Gut 62: 1806-1816, 2013.

11. Mantovani A, Valentino S, Gentile S, Inforzato A, Bottazzi B and Garlanda C: The long pentraxin PTX3: a paradigm for humoral pattern recognition molecules. Ann NY Acad Sci 1285: 1-14, 2013.

12. Kunes P, Holubcova Z, Kolackova $M$ and Krejsek J: Pentraxin 3(PTX 3): an endogenous modulator of the inflammatory response. Mediators Inflamm 2012: 920517, 2012.

13. Kusnierz-Cabala B, Gurda-Duda A, Dumnicka P, Panek J, Pawlica-Gosiewska D, Kulig J and Solnica B: Plasma pentraxin 3 concentrations in patients with acute pancreatitis. Clin Lab 59: 1003-1008, 2013.

14. Bottazzi B, Doni A, Garlanda C and Mantovani A: An integrated view of humoral innate immunity: pentraxins as a paradigm. Annu Rev Immunol 28: 157-183, 2010.

15. Sjöberg B, Qureshi AR, Anderstam B, Alvestrand A and Bárány P: Pentraxin 3, a sensitive early marker of hemodialysis-induced inflammation. Blood Purif 34: 290- 297, 2012.

16. Lin Q, Fu F, Shen L and Zhu B: Pentraxin 3 in the assessment of ventilator-associated pneumonia: an early marker of severity. Heart Lung 42: 139-145, 2013.

17. Hudzik B, Szkodzinski J, Pietka-Rzycka A, et al: Plasma pentraxin 3 may be a more sensitive marker of inflammatory response than high-sensitivity C-reactive protein after bare-metal stent compared to drug-eluting stent implantation. J Interferon Cytokine Res 33: 280-284, 2013. 